\title{
Resistivity Saturation in Metallic Liquids Above a Dynamical Crossover Temperature Observed in Measurements Aboard the International Space Station
}

\author{
D. C. Van Hoesen $\odot,{ }^{1}$ A. K. Gangopadhyay $\odot,{ }^{1, *}$ G. Lohöfer $\odot,{ }^{2}$ M. E. Sellers, ${ }^{1}$ C. E. Pueblo, ${ }^{1}$ \\ S. Koch, ${ }^{3}$ P. K. Galenko, ${ }^{3}$ and K. F. Kelton $\circledast^{1}$ \\ ${ }^{1}$ Department of Physics and the Institute of Materials Science and Engineering, Washington University in St. Louis, \\ St. Louis, Missouri 63130, USA \\ ${ }^{2}$ Institut für Materialphysik im Weltraum, Deutsches Zentrum für Luft- und Raumfahrt (DLR), 51170 Köln, Germany \\ ${ }^{3}$ Otto-Schott-Institut für Materialforschung, Friedrich-Schiller-Universität Jena, 07743 Jena, Germany
}

(Received 6 September 2019; revised manuscript received 23 October 2019; published 26 November 2019)

\begin{abstract}
Although a resistivity saturation (minimum conductivity) is often observed in disordered metallic solids, such phenomena in the corresponding liquids are not known. Here we report a saturation of the electrical resistivity in $\mathrm{Zr}_{64} \mathrm{Ni}_{36}$ and $\mathrm{Cu}_{50} \mathrm{Zr}_{50}$ liquids above a dynamical crossover temperature for the viscosity $\left(T_{A}\right)$. The measurements were made for the levitated liquids under the microgravity conditions of the International Space Station. Based on recent molecular dynamics simulations, the saturation is likely due to the ineffectiveness of electron-phonon scattering above $T_{A}$ when the phonon lifetime becomes too short compared to the electron relaxation time. This is different from the conventional resistivity saturation mechanisms in solids.
\end{abstract}

DOI: 10.1103/PhysRevLett.123.226601

Electron transport in metals is influenced by disorder, electron-electron correlation, and various elastic and inelastic scattering processes. While the effect of disorder is usually temperature independent (except for temperature induced defect formation), the characteristic energy scales with respect to temperature determine the relative importance of the various temperature-dependent scattering processes. The electrical resistivity usually increases with disorder and temperature. Therefore, with a sufficient amount of disorder and high thermal energy (temperature) it may saturate at high temperatures, reaching a minimum conductivity, when the mean free path becomes comparable to the interatomic spacings $(l \sim d)$, as was suggested by Ioffee and Regel [1] and Mott [2]. Most solids melt before this condition can be reached. However, many examples of resistivity saturation exist in disordered solids and glasses at low temperatures [3], A-15 [4] and Chevrel-phase [5] superconductors in the normal state, and heavy-fermion compounds [6]. There are also some indications for an approach to saturation in a few refractory high-melting temperature elemental solids [7]. In contrast, many crystalline metallic systems, the so-called "bad metals" (e.g., high- $T_{C}$ oxides [7,8], quantum-critical systems [9]), show no evidence for resistivity saturation even after attaining much higher values (in some cases up to a factor of 10) than the Ioffee-Regel-Mott (IRM) limit. The mechanisms responsible for this behavior in these exotic materials are currently under debate $[9,10]$ and are outside the scope of this work.

Here, we demonstrate the saturation of the resistivity at high temperature in two marginal glass-forming metallic liquids, $\mathrm{Zr}_{64} \mathrm{Ni}_{36}$ and $\mathrm{Cu}_{50} \mathrm{Zr}_{50}$. Interestingly, the saturation occurs just above the dynamical crossover temperature $T_{A}$, which is determined as the temperature at which the viscosity changes from an Arrhenius $\left\{\exp \left[-\left(E / k_{B} T\right)\right]\right\}$ behavior to a low temperature non-Arrhenius behavior, where the activation energy $E$ increases with decreasing temperature, $T\left(k_{B}\right.$ is the Boltzmann constant). The crossover temperature has become a subject of intense discussion in recent years [11-20]. Molecular dynamics simulations [11-14] have been particularly useful in elucidating the role of $T_{A}$. It has been related to the temperature-dependent lifetimes of the coordination numbers of local clusters, which begin to exceed the time required to communicate coordination changes to the neighboring atoms below this temperature. This communicated information sets the stage for correlated motion of atoms below $T_{A}$, which becomes increasingly more cooperative until glass formation. This suggests a natural link between liquid structure and dynamics. Since the resistivity is determined by structural disorder leading to elastic and inelastic scattering, the observation of a resistivity saturation above $T_{A}$ in the present case is taken as a clear demonstration of such a link between structure and liquid dynamics. Unlike the case in crystals and glasses, the saturation does not correspond to the largest electrical resistivity; the resistivities of the corresponding glasses are much larger. The behavior in the liquids, then, differs remarkably from that observed in conventional crystalline metallic crystals and glasses.

A direct link between structural changes at $T_{A}$ with the liquid dynamics has not been convincingly established 

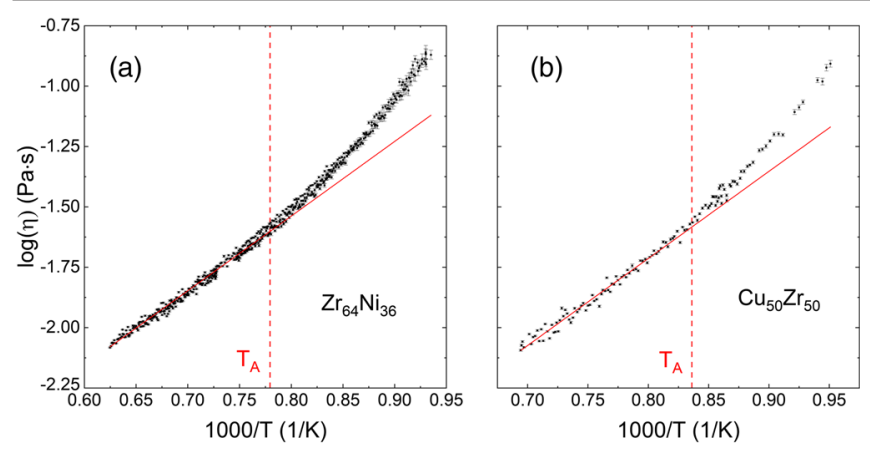

FIG. 1. The liquid shear viscosity for (a) $\mathrm{Zr}_{64} \mathrm{Ni}_{36}$ and (b) $\mathrm{Cu}_{50} \mathrm{Zr}_{50}$ liquids including the crossover temperatures $\left(T_{A}\right)$, measured by the oscillating drop technique on electrostatically levitated samples.

from experiments thus far. Some evidence has been reported in the static structure factor, $S(q)$ [21]. However, while correlated, the value of $T_{A}$ that is determined from the viscosity is larger than from the structural data. The overlap of the many different partial structure factors that contribute to the total $S(q)$ and the simultaneous presence of many different types of order in a liquid [22] likely obscure the onset of cooperativity in the $S(q)$ data. Moreover, as the lifetime of the coordination numbers or atomic bonds become too short above $T_{A}[13,14]$, the static structure factor may become insensitive to such changes. Electron scattering is very sensitive to static and rapidly changing chemical and structural order or disorder. Therefore, electrical transport (resistivity) measurements are routinely used to study order-disorder transformations in solids [23]. Naturally then, electrical resistivity is well suited to study rapid structural changes over small length scales, such as in the onset of cooperativity.

With the above objectives, the electrical resistivity was measured in the equilibrium and supercooled (i.e., below the liquidus temperature $T_{l}$ ) liquids of two marginal glassforming alloys, $\mathrm{Cu}_{50} \mathrm{Zr}_{50}$ and $\mathrm{Zr}_{64} \mathrm{Ni}_{36}$, in the microgravity environment of the International Space Station (ISS) using the electromagnetic levitation (EML) facility. The viscosities of equilibrium and supercooled liquid droplets ( $2.5 \mathrm{~mm}$ diameter) were measured under terrestrial conditions using our electrostatic levitation (ESL) facility [24] and the oscillating drop technique [25]. They are in agreement with measurements made aboard the ISS under microgravity [26] and in parabolic flight experiments [27]. However, because of higher resolution, data from the ground-based studies [16] were used for the determination of $T_{A}$. As shown in Fig. 1, $T_{A}$ is identified from the onset of deviation of the high temperature viscosity from a linear $\log (\eta)$ vs $T^{-1}$ plot. A statistical method [21] was used to give the most objective values of $T_{A}$ as $1283 \mathrm{~K} \pm 19 \mathrm{~K}$ for $\mathrm{Zr}_{64} \mathrm{Ni}_{36}$ and $1196 \mathrm{~K} \pm 21 \mathrm{~K}$ for $\mathrm{Cu}_{50} \mathrm{Zr}_{50}$, with a twosigma error.

Since an unconventional technique was used for the electrical resistivity measurements, a brief description of the facility and the experimental procedures are provided below; details may be found elsewhere [28]. Conventional four-probe techniques for the measurement of high melting-temperature liquids face major challenges due to contamination from the container and chemical reactions with probes and sample atmosphere, which are absent in the EML (containerless) processing under high vacuum. EML also enables studies of supercooled metastable liquids below $T_{l}$. However, EML studies under terrestrial conditions require large radio-frequency (rf) generated magnetic fields to levitate metallic samples. This produces large eddy currents that heat and melt the samples, preventing studies of supercooled liquids. While it is possible to bypass this problem by processing in $\mathrm{He}$ atmosphere, even the highest purity commercially available gases often contaminate the sample. Further, the strong magnetic forces produce turbulent flow in the liquid, which can disturb the measurements. These issues are alleviated using EML processing in a microgravity environment.

The MSL-EML facility aboard the ISS consists of watercooled $\mathrm{Cu}$ coils that generate two rf magnetic fields: (i) a quadrupole field that provides sample positioning and (ii) a superposed homogeneous dipole field to provide independent inductive sample heating (Fig. 2). The homogeneous rf field allows noninvasive, inductive measurements of the electrical resistivities [28] of the levitated solids and equilibrium and supercooled liquids. The decoupled heating and levitation allows the ISS/EML facility to be used to measure other thermophysical properties of metallic liquids, such as the specific heat [29]. The measurements are made on $6.5-8 \mathrm{~mm}$ diameter spherical samples.

As shown in Fig. 2, the sample is inductively coupled by the rf magnetic heating field to the resonant heating circuit, which is powered by a $400 \mathrm{kHz}$ rf power amplifier. The total complex admittance of the electrical heating circuit is

$$
\tilde{Y}_{\text {tot }}=2 i \omega C+\frac{2}{R_{L}+i \omega L+\tilde{Z}_{S}(a, \rho) / 2},
$$

where $i$ denotes the imaginary unit, $C$ is the condenser capacitance, $L$ is the coil inductance, and $R_{L}$ is the coil resistance. $\tilde{Z}_{S}(a, \rho)$ is the complex impedance of the sample, which depends on the sample radius $a$ and the electrical resistivity $\rho$. To obtain the total admittance of the circuit, defined by

$$
\tilde{Y}_{\mathrm{tot}}=\frac{I_{0}}{U_{o}} e^{-i \varphi},
$$

the sample coupling electronics (SCE) in the EML facility measures the amplitude of the rf current through the circuit $I_{o}$, the voltage drop over the circuit $U_{o}$, and the phase shift between the voltage and current $\varphi$ at a frequency $\omega$. Without a sample $\left(\tilde{Z}_{S}=0\right)$, measurements of these quantities enable the determination of the circuit parameters $C$, 


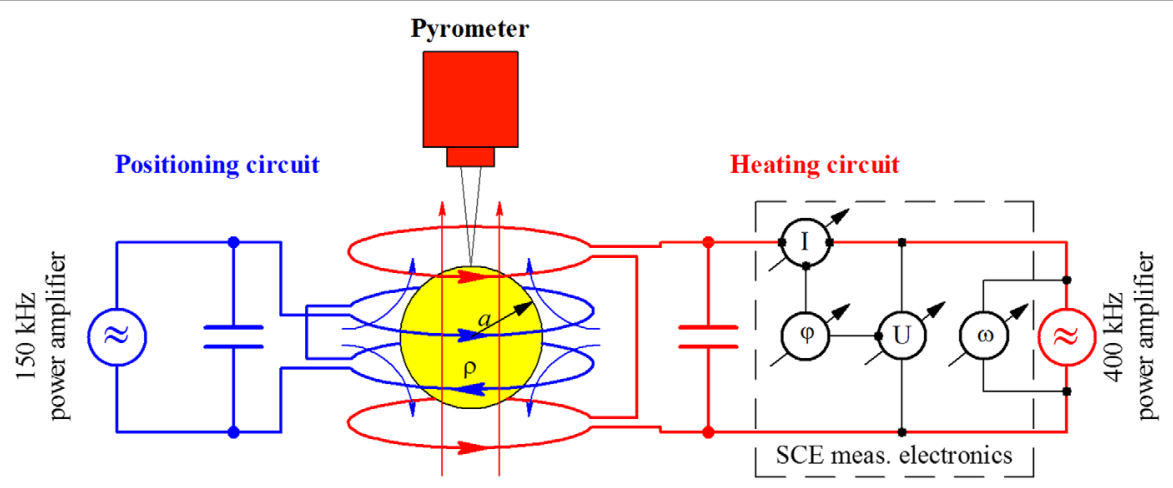

FIG. 2. A sample (yellow) at the center of the positioning and heating coils. A rf current in the opposite direction (left circuit) through the upper and lower coil generates a magnetic quadrupole field for the positioning of the sample. A rf current in the same direction through the coil generates a magnetic dipole field for heating the sample (right circuit). Also shown are the sample coupling electronics (SCE) for measuring the electrical admittance and a pyrometer for sample temperature measurement.

$L$, and $R_{L}$ from Eq. (1). A subsequent measurement of $\tilde{Y}_{\text {tot }}$ with a sample in the levitator yields the sample impedance $\tilde{Z}_{S}(a, \rho)$. For a spherical sample (as is the case for the liquid under microgravity) in a homogeneous rf magnetic field, the theoretical relation between $\tilde{Z}_{S}(a, \rho)$, the sample radius $a$, and the resistivity $\rho$, has been calculated in Ref. [30]. The temperature dependent sample radii, necessary for resistivity measurements, were determined from the video images of the levitated droplets using standard procedures [31,32]. The experimental method and the measured specific volumes of the liquids are presented in S. 2 in the Supplemental Material [33].

In a typical measurement cycle, the sample is levitated and then melted by increasing the current through the heater coils. The heater and positioner currents are then reduced and kept constant during cooling throughout the resistivity measurement. Figure 3 shows the resistivity data for both liquids during two representative thermal cycles. In some cases, small cycle-to-cycle variations $(<1 \%)$ in the absolute magnitude of resistivity were observed. This could
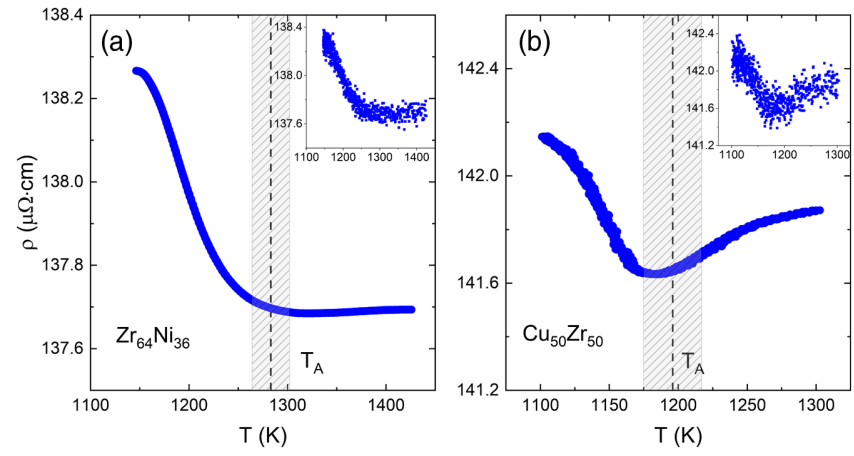

FIG. 3. The electrical resistivity, smoothed by 200 point averaging, as a function of temperature in liquid (a) $\mathrm{Zr}_{64} \mathrm{Ni}_{36}$ and (b) $\mathrm{Cu}_{50} \mathrm{Zr}_{50}$, showing near saturation at or above $T_{A}$. The shaded regions represent the uncertainties in $T_{A}$. The original data are shown in the insets. be due to small changes in the sample position relative to the coil and/or small changes in temperature of the measurement electronics. Considering this and the precision in radius measurements $(1 \%$ in absolute magnitude and $0.01 \%$ in relative changes, see S. 2 in Ref. [33]), the error in the absolute resistivity would be close to $2 \%$ and relative changes to about $0.7 \%$. However, the temperature dependence of the resistivity remained the same in all measurement cycles. For both alloys, the temperature coefficients of the resistivity $(d \ln \rho / d T)$ are negative, which are usually observed in high resistivity metallic glasses $[3,34]$. The most interesting observation is the near saturation of resistivity above the crossover temperature $T_{A}$, determined from the viscosity measurements. We are not aware of any previous report demonstrating the saturation of the resistivity in a metallic liquid. While the resistivity of $\mathrm{Zr}_{64} \mathrm{Ni}_{36}$ saturates precisely at $T_{A}$, the $\mathrm{Cu}_{50} \mathrm{Zr}_{50}$ resistivity goes through a minimum around $T_{A}$ before saturating at a higher temperature. The reason for this slightly different behavior for the $\mathrm{Cu}_{50} \mathrm{Zr}_{50}$ liquid is currently unclear.

The sign of $d \ln \rho / d T$ depends very much on the magnitude of the resistivity of the alloy, as was observed by Mooij [34] quite a while ago. A sign change from positive to negative coefficient was observed around $150 \mu \Omega \mathrm{cm}$, although this was later found to be not universal [35]. This may be explained in many different ways (see Ref. [3] and [36]). The pseudopotential based Ziman theory [37-39] or its extension, the $t$ matrix based Ziman theory for the element [40] and alloy [41] liquids, are some of these. Both approaches express the resistivity in terms of the structure factor, $S(q)$ [partial $S(q) s$ for the alloys] and the pseudopotential $|V(q)|[37,38]$, or the scattering matrix ( $t$ matrix) [40,41]. In both cases, the temperature dependence of resistivity arises from the change in the $S(q)$ with temperature, since the pseudopotentials or the $t$ matrix are temperature independent in these theories. When the Fermi wave vector $2 k_{F}$ lies on the higher $q$ side of the first peak of $S(q), d \ln \rho / d T$ becomes 
negative because of the decrease of $S\left(q_{2 k_{F}}\right)$ with increasing temperature. Using the liquid partial structure factors from the molecular dynamics (MD) simulations of $\mathrm{Zr}_{64} \mathrm{Ni}_{36}$ and $\mathrm{Cu}_{50} \mathrm{Zr}_{50}$ and reasonable values for $k_{F}$, negative temperature coefficients of resistivities for both alloys could be reproduced (see S.1 in the Supplemental Material [33], which includes Refs. [37-43]). A quantitative agreement with experiments is not expected, since such calculations are very sensitive to the choice of $E_{F}$ and $k_{F}$ [44]. However, most importantly, while the negative $d \ln \rho / d T$ can be qualitatively explained by the Faber-Ziman type theories, the saturation of resistivity above $T_{A}$ cannot be because the $S(q)$ and its partials continue to decrease at nearly the same rates above and below $T_{A}[45,46]$.

Given that the $\mathrm{Zr}_{64} \mathrm{Ni}_{36}$ and $\mathrm{Cu}_{50} \mathrm{Zr}_{50}$ liquids contain valence electrons coming from both $s$ - and $d$-electron shells, Mott's [47] idea of scattering of $s$ electrons into the partially empty $d$ bands due to fluctuations of atomic separations in disordered and liquid alloys may also be relevant. The probability of an $s-d$ transitions in this model does not explicitly depend on the structure factor, but on the available empty states near the Fermi level, $N\left(E_{F}\right)$. Since the density of states is weakly temperature dependent, if $N\left(E_{F}\right)$ lies on the higher energy side of a maximum, the probability of $s-d$ transitions may show a small decrease, and therefore a small negative $d \ln \rho / d T$, as observed for the two liquids. However, a saturation at high temperatures is not expected.

Electron transport theories are typically based on the Boltzmann equation, which treats electrons in between collisions as classical particles. However, when the mean free path approaches the IRM limit, interference of the incident and scattered electron waves become important and the Boltzmann transport theory must be modified. This is called the "weak or incipient electron localization" regime, which occurs when there is sufficient quantum interference of the incident and scattered waves [48-50]. The idea is similar to those developed by Anderson for electron localization in disordered solids [51]. However, instead of conduction by hopping with increasing temperature in Anderson localization, the increase in conductivity for weak localization is due to the dephasing (loss of coherence) of the scattered waves from inelastic scattering by phonons, which weakens localization. The conductivity $\sigma$ then rises linearly with temperature far below the Debye temperature $\theta_{D}$ and as $\sqrt{ } T$ above it $[49,50]$.

To check this, the resistivities of the corresponding glasses were measured by a commercial PPMS (Physical Property Measurement System, Quantum Design, CA) at low temperatures. $2 \mathrm{~mm}$ wide and $20 \mu \mathrm{m}$ thick amorphous ribbons, produced by the conventional melt-quench technique, were used for this purpose. Figure 4 shows the conductivity (not the resistivity) of both glasses and liquids of $\mathrm{Zr}_{64} \mathrm{Ni}_{36}$ and $\mathrm{Cu}_{50} \mathrm{Zr}_{50}$. In the glass at temperatures above approximately $100 \mathrm{~K}, \sigma \propto \sqrt{ } T$, which is consistent

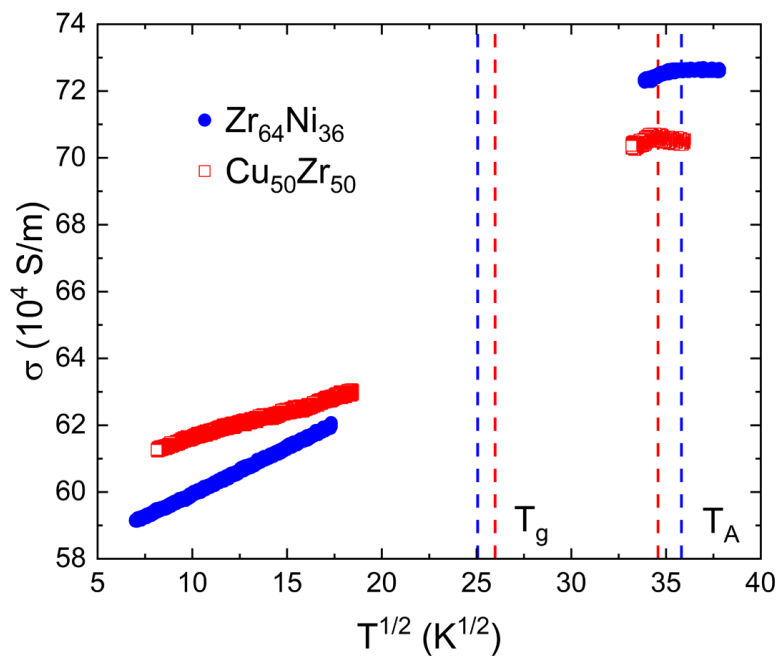

FIG. 4. The electrical conductivities of glassy and liquid $\mathrm{Zr}_{64} \mathrm{Ni}_{36}$ and $\mathrm{Cu}_{50} \mathrm{Zr}_{50}$. The glass transition $\left(T_{g}\right)$ and crossover $\left(T_{A}\right)$ temperatures are also shown.

with weak localization theories. A change to $\sigma \propto T$ at the lowest temperatures $\left(T \ll \theta_{D}\right)$ is also expected in the same theoretical framework, which was observed by Howson and Grieg [50] in the $\mathrm{Cu}_{50} \mathrm{Zr}_{50}$ glass. Because of the limited amount of data for the liquid at temperatures below $T_{A}$, it is difficult to state clearly whether the liquid follows the same functional relationship with temperature as the glass at high temperatures. However, it is clear from Fig. 3 that the temperature dependence is nonlinear.

The local order in a glass below $T_{g}$ does not change with temperature (except for very slow structural relaxation) and the increase in conductivity is entirely due to decreasing elastic or inelastic scattering. In contrast, above $T_{g}$ in the supercooled liquid and above $T_{l}$ in the equilibrium liquid, the spatial and temporal SRO changes continuously. This is expected to change the active high-frequency phonons responsible for the inelastic scattering of electrons. Such changes in phonon scattering are not considered in the weak localization theories.

Since no theory for "weak localization" for systems with changing short or medium range order is available, it is not yet possible to quantitatively understand the mechanism responsible for the saturation of the resistivity in the liquid. However, we put forward a qualitative explanation. Saturation above $T_{A}$ for both liquids is a strong indication that it is related to the liquid structure. It is well known that the spatial and temporal changes of the liquid structure (structural relaxations) are strongly temperature dependent. The timescales for structural relaxation change from about $100 \mathrm{~s}$ near $T_{g}$ to $10^{-12}-10^{-14} \mathrm{~s}$ in the equilibrium liquids $[12-14,52]$. Since the typical scattering time for electrons is in the nano- to femtoseconds range, the liquid structure appears as static to the electrons in most of the supercooled states. It is, therefore, reasonable to assume that the resistivity or conductivity changes in this temperature 
range are mostly due to electron-phonon scattering. Although liquids cannot sustain long wavelength phonons, short wavelength (high frequency) phonons are excited in short-lived solidlike regions, as suggested by Frenkel [53], and has been observed in inelastic scattering experiments [54]. With increasing temperature, the length scales and timescales of the dynamically evolving solidlike regions (atomic clusters) in a liquid decrease [12-14,46,52]. Above some temperature, the mean electron scattering time and the structural relaxation time may become comparable. The effectiveness of electron-phonon scattering will also depend on the relative timescales of the phonon frequencies and the electron scattering times. Therefore, above some characteristic temperature, the scattering of electrons by structural disorder and phonon scattering will become ineffective, resulting in a saturation of the electrical resistivity or conductivity. It is reasonable that this temperature appears to be $T_{A}$, since above this temperature the lifetime of a local cluster is too short to communicate this information to neighboring atoms and the phonons become effectively localized [11]. Since the dynamical crossover is observed in all liquids $[11,18,55]$, except for the very strong ones, resistivity saturation may be a universal property of liquids. That the saturation coincides with $T_{A}$ is perhaps the most direct evidence that the structure of the liquid at the atomic level strongly couples to the dynamics at a longer, hydrodynamic, level [52].

Using the nearly free electron theory, it is possible to estimate the electron mean free path in the liquids. Assuming effective valences (2.43 for $\mathrm{Cu}_{50} \mathrm{Zr}_{50}$ and 2.77 for $\mathrm{Zr}_{64} \mathrm{Ni}_{36}$, see S. 1 [33]), Fermi wave vectors $\left(k_{f}=1.40\right.$ and $1.52 \AA^{-1}$ for $\mathrm{Cu}_{50} \mathrm{Zr}_{50}$ and $\mathrm{Zr}_{64} \mathrm{Ni}_{36}$, respectively, see S.1 [33]), and molar volumes $\left(10.99 \times 10^{-6}\right.$ and $11.54 \times$ $10^{-6} \mathrm{~m}^{3}$ for $\mathrm{Cu}_{50} \mathrm{Zr}_{50}$ and $\mathrm{Zr}_{64} \mathrm{Ni}_{36}$, respectively, see S.2 [33]), these are 3.04 for $\mathrm{Cu}_{50} \mathrm{Zr}_{50}$ and $3.14 \AA$ for $\mathrm{Zr}_{64} \mathrm{Ni}_{36}$. They are close to the positions of the first maximum in the experimentally determined pair correlation functions (2.81 for $\mathrm{Cu}_{50} \mathrm{Zr}_{50}$ and $3.12 \AA$ for $\mathrm{Zr}_{64} \mathrm{Ni}_{36}$ ) [56], which supports the idea that the mean-free path is close to the interatomic spacing in the liquids. This work also demonstrates that electrical transport, in lieu of dynamical properties, can be used as a powerful tool to determine $T_{A}$ and the local order in liquids. Finally, it should be emphasized that such contamination-free precision measurements were only possible by a unique combination of the containerless processing technique, a novel measurement technique, and the microgravity environment of the International Space Station.

This work was partially supported by NASA under Grants No. NNX10 AU19G and No. NNX16AB52G as well as the German Aerospace Center (DLR), Space Administration under Contract No. 50WM1541. The authors acknowledge the access to the ISS/EML, which is a joint undertaking of the European Space Agency (ESA) and the DLR Space Administration. The reported work was conducted in the framework of the ESA research projects: Electrical Resistivity (AO-2000-038), MULTIPHAS (AO-2004-114) and QUASI (AO-2009-0959). The experiments were operated from the "Microgravity User Support Center" (MUSC) at the Deutsches Zentrum für Luft- und Raumfahrt (DLR) in Cologne, Germany. The authors express their gratitude to the members of the MUSC for their cooperation and assistance throughout the inception, planning, and execution of the experiments in the ISS/EML facility onboard the International Space Station. Any opinions, findings, and conclusions or recommendations expressed in this material are those of the author(s) and do not necessarily reflect the views of NASA.

*Corresponding author. anup@wuphys.wustl.edu

[1] A. F. Ioffe and A. B. Regel, Prog. Semicond. 4, 237 (1960).

[2] N. F. Mott, Metal-Insulator Transitions (Taylor and Francis, London, 1974), p. 23.

[3] M. A. Howson and B. L. Gallagher, Phys. Rep. 170, 265 (1988).

[4] Z. Fisk and G. W. Webb, Phys. Rev. Lett. 36, 1084 (1976).

[5] C. S. Sunandana, J. Phys. C 12, L165 (1979).

[6] A. de Visser, J. J. M. Franse, and A. Menovsky, J. Magn. Magn. Mater. 43, 43 (1984).

[7] O. Gunnarsson, M. Calandra, and J. E. Han, Rev. Mod. Phys. 75, 1085 (2003).

[8] R. A. Cooper, Y. Wang, B. Vignolle, O. J. Lipscombe, S. M. Hayden, Y. Tanabe, T. Adachi, Y. Koike, M. Nohara, H. Takagi, Cyril Proust, and N. E. Hussey, Science 323, 603 (2009).

[9] S. Sachdev, Quantum Phase Transitions (Cambridge University Press, New York, 1999).

[10] S. A. Hartnoll, Nat. Phys. 11, 54 (2015).

[11] A. Jaiswal, T. Egami, K. F. Kelton, K. S. Schweizer, and Y. Zhang, Phys. Rev. Lett. 117, 205701 (2016).

[12] Y. Fan, T. Iwashita, and T. Egami, Phys. Rev. Lett. 115, 045501 (2015).

[13] T. Iwashita, D. M. Nicholson, and T. Egami, Phys. Rev. Lett. 110, 205504 (2013).

[14] R. Soklaski, Z. Nussinov, Z. Markow, K. F. Kelton, and L. Yang, Phys. Rev. B 87, 184203 (2013).

[15] Y. C. Hu, F. X. Li, M. Z. Li, H. Y. Bai, and W. H. Wang, J. Appl. Phys. 119, 205108 (2016).

[16] M. E. Blodgett, T. Egami, Z. Nussinov, and K. F. Kelton, Sci. Rep. 5, 13837 (2015).

[17] B. Schmidtke, N. Petzold, R. Kahlau, and E. A. Rössler, J. Chem. Phys. 139, 084504 (2013).

[18] F. Mallamace, C. Branca, C. Corsaro, N. Leone, J. Spooren, S. Chen, and H. E. Stanley, Proc. Natl. Acad. Sci. U.S.A. 107, 22457 (2010).

[19] J. P. Garrahan and D. Chandler, Proc. Natl. Acad. Sci. U.S.A. 100, 9710 (2003).

[20] S. Sastry, P. G. Debenedetti, and F. H. Stillinger, Nature (London) 393, 554 (1998).

[21] R. Dai, R. Ashcraft, and K. F. Kelton, J. Chem. Phys. 148, 204502 (2018). 
[22] A. K. Gangopadhyay and K. F. Kelton, J. Chem. Phys. 148, 204509 (2018).

[23] L. W. Shacklette and W. S. Williams, Phys. Rev. B 7, 5041 (1973).

[24] N. Mauro and K. F. Kelton, Rev. Sci. Instrum. 82, 035114 (2011).

[25] W. K. Rhim, K. Ohsaka, P. F. Paradis, and R. Erik Spjut, Rev. Sci. Instrum. 70, 2796 (1999).

[26] M. Mohr, R. K. Wunderlich, S. Koch, P. K. Galenko, A. K. Gangopadhyay, K. F. Kelton, J. Z. Jiang, and H.-J. Fecht, Microgravity Sci. Technol. 31, 177 (2019).

[27] P. Heintzmann, F. Yang, S. Schneider, G. Lohöfer, and A. Meyer, Appl. Phys. Lett. 108, 241908 (2016).

[28] G. Lohöfer, Rev. Sci. Instrum. 89, 124709 (2018).

[29] R. K. Wunderlich, H.-J. Fecht, and R. Willnecker, Appl. Phys. Lett. 62, 3111 (1993).

[30] G. Lohöfer, Int. J. Eng. Sci. 32, 107 (1994).

[31] W. K. Rhim, S. K. Chung, D. Barber, K. F. Man, G. Gutt, A. Rulison, and R. E. Spjut, Rev. Sci. Instrum. 64, 2961 (1993).

[32] R. C. Bradshaw, D. P. Schmidt, J. R. Rogers, K. F. Kelton, and R. W. Hyers, Rev. Sci. Instrum. 76, 125108 (2005).

[33] See Supplemental Material at http://link.aps.org/ supplemental/10.1103/PhysRevLett.123.226601 for the estimation of temperature coefficients of resistivity and experimentally measured sample volumes.

[34] J. H. Mooij, Phys. Status Solidi A 17, 521 (1973).

[35] C. C. Tsuei, Phys. Rev. Lett. 57, 1943 (1986).

[36] P. A. Lee and T. V. Ramakrishnan, Rev. Mod. Phys. 57, 287 (1985).

[37] J. M. Ziman, Philos. Mag. 6, 1013 (1961).

[38] T. E. Faber and J. M. Ziman, Philos. Mag. 11, 153 (1965).

[39] T. E. Faber, Introduction to the Theory of Liquid Metals (Cambridge University Press, Cambridge, England, 1972).
[40] R. Evans, D. A. Greenwood, and P. Lloyd, Phys. Lett. A 35, 57 (1971).

[41] O. Dreirach, R. Evans, H.-J. Guntherodt, and H.-U. Kunzi, J. Phys. F 2, 709 (1972).

[42] J. K. Baria and A. R. Jani, Phys. Rev. B 328, 317 (2003).

[43] R. Taylor, J. Phys. F 8, 1699 (1978).

[44] E. Esposito, H. Ehrenreich, and C. D. Gelatt, Jr., Phys. Rev. B 18, 3913 (1978).

[45] A. K. Gangopadhyay, M. E. Blodgett, M. L. Johnson, A. J. Vogt, N. A. Mauro, and K. F. Kelton, Appl. Phys. Lett. 104, 191907 (2014).

[46] A. K. Gangopadhyay, C. E. Pueblo, R. Dai, M. L. Johnson, R. Ashcraft, D. Van Hoesen, M. Sellers, and K. F. Kelton, J. Chem. Phys. 146, 154506 (2017).

[47] N. F. Mott, Philos. Mag. 26, 1249 (1972).

[48] Y. Imry, Phys. Rev. Lett. 40, 469 (1978).

[49] M. A. Howson, J. Phys. F 14, L25 (1984).

[50] M. A. Howson and D. Greig, J. Phys. F 16, 989 (1986).

[51] P. W. Anderson, Phys. Rev. 109, 1492 (1958).

[52] R. Ashcraft, Z. Wang, D. L. Abernathy, D. G. Quirinale, T. Egami, and K. F. Kelton, arXiv:1810.02351.

[53] J. Frenkel, Kinetic Theory of Liquids, edited by R. H. Fowler, P. Kapitza, and N. F. Mott (Oxford University Press, New York, 1947).

[54] E. Burkel, Rep. Prog. Phys. 63, 171 (2000).

[55] A. K. Gangopadhyay and K. F. Kelton, J. Mater. Res. 32, 2638 (2017).

[56] A. K. Gangopadhyay, M. E. Blodgett, M. L. Johnson, J. McKnight, V. Wessels, A. J. Vogt, N. A. Mauro, J. C. Bendert, R. Soklaski, L. Yang, and K. F. Kelton, J. Chem. Phys. 140, 044505 (2014). 